\title{
Central Contributions of Myocardial Injury to Adverse Outcomes in Patients With Coronavirus Disease 2019
}

Hu Tan

Army Medical University

Chuan Liu

Army Medical University

Jie Yang

Army Medical University

Yuanqi Yang

Army Medical University

Yang Shen

Army Medical University

Renzheng Chen

Army Medical University

Xiaohan Ding

People's Liberation Army Joint Logistic Support Force 940th Hospital

Xubin Gao

Army Medical University

Jingbin Ke

Army Medical University

Fangzhengyuan Yuan

Army Medical University

Chunyan $\mathrm{He}$

Army Medical University

Qi Li

Army Medical University

Mingdong $\mathrm{Hu}$

Army Medical University

Limin Zhang

Army Medical University

Ping Li

Army Medical University

Lan Huang ( $\square$ huanglan260@126.com )

Institute of Cardiovascular Diseases of PLA, the Second Affiliated Hospital, Army Medical University, Chongqing, China; https://orcid.org/0000-0001-62002309

\section{Research}

Keywords: COVID-19, Severe case, Myocardial injury, Risk factors, Clinical outcomes

Posted Date: November 2nd, 2020

DOI: https://doi.org/10.21203/rs.3.rs-99444/v1

License: (c) (i) This work is licensed under a Creative Commons Attribution 4.0 International License. Read Full License 


\section{Abstract}

Objective: this study aimed to explore the impacts of myocardial injury on the clinical severity and outcomes in patients with Coronavirus Disease 2019 (COVID-19).

Methods: we analyzed the electronic medical records of 1646 COVID-19 inpatients in Wuhan Huoshenshan Hospital.

Results: 327 (19.9\%) developed into severe cases, 23 (1.4\%) died. In comparison to common cases, severe cases showed older age, more comorbidities, abnormal immune responses, as well as liver, renal, cardiac and coagulation disorders. Multivariable logistic regression identified that older age , combining with arrhythmia, abnormal lymphocyte percentage, elevated hypersensitive $\mathrm{C}$ reactive protein (hs-CRP) and myocardial injury were the independent risk factors for the incidence of severe cases. Moreover, Kaplan-Meier survival analysis showed that patients with myocardial injury had increasing risks of mortality, incidence of severe cases, acute respiratory distress syndrome (ARDS), and intensive care unit (ICU) admission. Particularly, myocardial injury patients co-existed with any other risk factor further deteriorated the clinical outcomes.

Conclusion: The presence of myocardial injury and its co-existing with older age, arrhythmia, abnormal lymphocyte percentage, or elevated hs-CRP were greatly associated with the incidence of severe patients and poor clinical outcomes.

\section{Introduction}

Coronavirus disease 2019 (COVID-19) is a current pandemic infection caused by severe acute respiratory syndrome coronavirus 2 (SARS-CoV-2), which has raised great concerns all over the world. At present, more than 200 countries and/or districts have confirmed more than two million cases infected with SARS-CoV-2, and the number is still rising. Direct and indirect contacting with mucous membranes in the nose, mouth, or eyes is considered as the predominant transmitted pathways [1]. SARS-CoV-2 infected patients present series of clinical symptoms including fever, cough, myalgia, fatigue, and/or dyspnea, et al [2]. Many patients showed bilateral involvement of chest CT images and laboratory examination showed pronounced lymphopenia [3, 4]. Moreover, growing data from COVID-19 patients identify the presence of myocardial injury in critically ill patients and/or deceased patients [2, 5], which has been proved to be associated with the fatal outcomes [6]. However, the impacts of myocardial injury on the clinical course and non-fatal outcomes are largely unknown.

During the progression of COVID-19 patients, severe patients are always linked to poor outcomes. Recent reports indicated that the percentage of severe cases in COVID-19 patients ranged from 15 to $30 \%$, and the severe patients were more likely to suffer from multiple organic dysfunctions, receive intensive care unit (ICU) care, ventilator therapy, and have a higher mortality rate [7-9]. Moreover, the severe patients posed great strain on medical resources, especially if they are not adequately staffed or resourced. Until now, evidence-based treatments are absent and supportive care is the mainstay of clinical management. Early recognition of severe patients may be beneficial to reasonable triage to the critical care managements and improve our understanding of the clinical course of COVID-19. Considering that severe cases usually share similar clinical features with critically ill patients, including myocardial injury [10], we hypothesize that myocardial injury may contribute to the incidence of severe COVID-19 cases and its clinical outcomes.

Accordingly, we conducted this large-scale retrospective cohort study to determine the risk factors for the incidence of severe COVID-19 cases and the impacts of myocardial injury on the clinical course and outcomes.

\section{Methods}

\section{Study design and Participants}

This single-centre, retrospective cohort study was performed at Huoshenshan Hospital (Wuhan, China), which is an urgently constructed hospital for the diagnosis and management of COVID-19 patients. Adult inpatients ( $\geq 18$ years old) were consecutively enrolled from February $4^{\text {th }}, 2020$ to March $9^{\text {th }}, 2020$ throughout Wuhan without any other selectivity. All the patients were diagnosed with COVID-19 according to WHO interim guidance [11]. This study was approved by the Human Ethics Committee, Huoshenshan Hospital (NO. HSSLL023) and conformed to the Declaration of Helsinki, oral consent was obtained from each patient when enrollment due to the rapid emergence of COVID-19.

\section{Data Collection}

All the data were obtained from standardized clinical electronic medical records, including demographic information, symptoms, signs, comorbidities, nursing records, laboratory tests, chest CT images, treatments, and outcomes. The time of illness onset was the day when the symptom was initially reported. The time from illness onset to normothermia, inflammatory resorption from CT images, viral shedding, the diagnosis of severe cases, respiratory failure, ARDS, ICU admission, and death were recorded. Hospital and ICU length of stay were calculated. All the data were carefully checked by two doctors (TH and YJ) and the third researcher (LC), who adjudicated any differences in interpretation between the two doctors.

\section{Laboratory examinations at admission}

Laboratory testings included routine blood test, renal and liver function, coagulation profile, myocardial enzymes, hypersensitive cardiac troponin I (hs-cTnl), hypersensitive $\mathrm{C}$ reactive protein (hs-CRP) and so on. Throat-swab specimens were collected and SARS-CoV-2 was detected by real time-PCR as previously described [5]. Chest CT scans were performed in all patients before or at admission and followed up during hospitalization. 
The clinical severity was defined according to the guidelines for diagnosis and management of COVID-19 ( $5^{\text {th }}$ version, in Chinese) released by National Health Commission of China [12]. Severe cases was defined when meeting any of the follows: respiratory distress, respiratory rate $\geq 30$ times/min; pulse oxygen saturation $\leq 93 \%$ at rest; arterial oxygen partial pressure/fraction of inspired oxygen $\leq 300 \mathrm{mmHg}$; more than $50 \%$ pulmonary lesion progression in chest CT images within 24 to 48 hours.

\section{Definitions}

The abnormalities in laboratory findings were based on the criteria in Huoshenshan hospital. Acute respiratory distress syndrome (ARDS) was defined according to the Berlin Definition [13]. Myocardial injury was defined if the circulating level of cardiac biomarkers (eg, hs-cTnl) were above the $99^{\text {th }}$ percentile upper reference limit [14]. Acute kidney injury was identified on the basis of serum creatinine over base 1.5 times [15]. Liver injury was diagnosed if the alanine aminotransferase (ALT) was above the upper limit of the reference range [16]. Coagulopathy was defined as a 3-second and 5-second extension of prothrombin time (PT) and activated partial thromboplastin time, respectively.

\section{Clinical outcomes}

The primary endpoints were mortality, the secondary endpoints included the incidence of severe cases, ARDS, receiving mechanical ventilation, ICU admission, as well as clinical cure and discharges. The criteria of discharges included absence of fever for $\geq 3$ days; obvious pulmonary inflammatory resorption in chest CT; clinical remission of respiratory symptoms; and two times of negative for SARS-CoV-2 RNA at least $24 \mathrm{~h}$ apart [6]. The clinical outcomes (mortality for 40 days and others for 60 days) were monitored after the initial symptom onset.

\section{Statistical analysis}

Continuous variables were presented as median (interquartile range [IQR]) and categorical variables were expressed as n (\%). Mann-Whitney U test, Pearson $\chi^{2}$ test or Fisher's exact test were employed to compare the differences between two groups as appropriate. To identify the risk factors for severe cases, univariable and multivariable binary logistic regression models were used. Considering to avoid overfitting in the model, variables were chosen for univariable analysis on the basis of previous findings and clinical constraints. We used the method of forward LR to conduct multivariable analysis ( $p<0.10$ for entry, $p<0.050$ for stay). Odds ratio (OR) was used for determining relation between potential risk factors and severe cases. $p<0.050$ was considered statistically significant. Kaplan-Meier curves and log-rank tests were used to compare survival rate and event-free survival rate. Statistical analyses were performed using the SPSS software 26.

\section{Results}

\section{Basic characteristics, clinical features, laboratory findings and outcomes in total patients}

In this study, from February $4^{\text {th }}$ to March $9^{\text {th }} 2020$, we enrolled 1646 COVID-19 confirmed patients, who hospitalized in Wuhan Huoshenshan Hospital. The median age was 60.0 years (IQR 50.0-68.0), 830 (50.4\%) patients were male, 93 (5.7\%) were current smoker and 56 (3.4\%) addicted to alcohol. The initial symptom included fever (1034 [62.8\%]), cough/sputum (277 [16.8\%]), fatigue (108 [6.6\%]) or short of breath (71 [4.3\%]). Gastrointestinal symptoms such as nausea, vomiting, diarrhea might occur in early as well. In addition, 48 patients (2.9\%) showed no significant discomfort. Hypertension (490 [29.8\%]) and diabetes (220 [13.4\%]) were the common comorbidities. $1312(79.7 \%)$ patients showed bilateral pneumonia while $133(8.1 \%)$ patients showed unilateral pneumonia, and 1482 (90.0\%) patients exhibited ground-glass opacity. COVID-19 patients showed elevated hs-CRP (2.7 [IQR 1.0-10.3] mg/L), the mean levels of other laboratory results in all patients were within the reference ranges. However, 294 (17.9\%), 19 (1.2\%), 117 (7.1\%) and 28 (1.7\%) patients suffered from liver injury, kidney injury, myocardial injury and coagulation disorder, respectively. (Table 1)

Most patients received antivirus therapy, including abidor hydrochloride (751 [45.6\%]), oseltamivir phosphate (194 [11.8\%]), and ribavirin (77 [4.7\%]). Moxifloxacin hydrochloride was used in 493 (30.0\%) patients to anti-infection. Vitamin C (481 [29.2\%]), thymalfasin (123 [7.5\%]) and recombinant interference (104 [6.3\%]) were used to regulate immune function. In addition, a small percentage of patients were treated with glucocorticoid (215 [13.1\%]).

The median hospital length of stay was 14.0 days (IQR 9.0-19.0), the median time from illness onset to normothermia was 24.0 days (IQR 17.0-32.0), the median time from illness onset to inflammatory resorption was 30.0 days (IQR 23.0-36.0), and the median time from illness onset to viral shedding was 29.0 days (IQR 24.0-36.0). 73 (4.4\%) subjects were complicated with ARDS, 57 (3.5\%) subjects progressed into respiratory failure, 60 (3.7\%) subjects were admitted to ICU, and $43(2.6 \%)$ subjects received mechanical ventilation. The median time from illness onset to severe illness was 20.0 days (IQR 14.0-26.0), the median time from illness onset to ARDS was 18.0 days (IQR 14.0-26.5), the median time from illness onset to respiratory failure was 18.5 days (IQR 14.0-25.0), and the median time from illness onset to use mechanical ventilation was 20.0 days (IQR 13.0-26.0). The median time from illness onset to ICU admission was 18.0 days (IQR 14.0-25.0) and the median ICU length of stay was 5.5 days (IQR 3.0-9.0). 23 (1.4\%) patients died during hospitalization, and the median time from illness onset to death was 26.0 days (IQR 17.0-31.0). (Table 2)

\section{Differences of basic characteristics, clinical features, laboratory findings and outcomes between common patients and severe patients}

327 (19.9\%) individuals developed into severe cases. Compared to common cases, severe patients showed older age (65.0 [IQR 56.0-72.0] vs 58.0 [IQR 48.067.0] years, $\mathrm{p}<0.0001)$, lower proportion of fever (184 [56.3\%] vs 850 [64.4\%], $\mathrm{p}=0.0062)$, on the contrary, higher proportion of short of breath (24 [7.3\%] vs 47 [3.6\%], $\mathrm{p}=0.0026)$, more comorbidities including hypertension (138 [42.2\%] vs 352 [26.7\%], $\mathrm{p}<0.0001)$, diabetes $(66$ [20.2\%] vs 154 [11.7\%], $\mathrm{p}<0.0001)$, arrhythmia (25 [7.6\%] vs 28 [2.1\%], p<0.0001), and malignant neoplasm (10 [3.1\%] vs 18 [1.4\%], $\mathrm{p}=0.034)$. 
In addition, severe patients exhibited higher leucocytes (6.7 [IQR 5.3-8.3] vs 5.7 [IQR 4.7-6.8] × 109/L, p<0.0001), neutrophil percentage (69.9 [IQR 62.6-78.0] vs 60.7 [IQR 54.8-66.5] \%, p<0.0001) but lower lymphocyte percentage (18.9 [IQR 12.9-26.1] vs 28.2 [IQR 23.0-33.6] \%, p<0.0001), monocyte percentage (7.6 [IQR 6.0-8.9] vs 7.7 [IQR 6.6-9.0] \%, p=0.010), eosinophil percentage (1.6 [IQR 0.9-2.6] vs 2.0 [IQR 1.3-3.1] \%, p<0.0001), basophil percentage (0.28 [IQR 0.150.40] vs 0.40 [IQR 0.28-0.50] \%, p<0.0001), and hemoglobin concentration (116.3 [IQR 104.5-127.7] vs 125.5 [IQR 116.0-136.0] g/L, p<0.0001). Particularly, the hs-CRP concentrations in severe patients were significantly higher (15.6 [IQR 4.5-45.0] vs 2.0 [IQR 0.8-5.9] mg/L, $\mathrm{p}<0.0001)$ than those in common patients. Moreover, compared to common cases, severe patients showed higher ALT (29.3 [IQR 17.6-50.4] vs 23.9 [IQR 15.3-38.9] IU/L, p<0.0001), aspartate aminotransferase (22.4 [IQR 18.3-32.5] vs 19.4 [IQR 15.6-25.7] IU/L, $\mathrm{p}<0.0001)$, total bilirubin (9.5 [IQR 7.2-12.4] vs 9.0 [IQR 7.2-11.5] $\mu \mathrm{mol} / \mathrm{L}, \mathrm{p}=0.044)$, gamma-glutamyl transpeptidase (37.0 [IQR 23.1-60.4] vs 30.2 [IQR 20.1-48.4] IU/L, p<0.0001), urea nitrogen (5.0 [IQR 4.0-6.5] vs 4.3 [IQR 3.6-5.2] mmol/L, $\mathrm{p}<0.0001)$, and cystatin C (1.0 [IQR 0.9-1.2] vs 0.9 [IQR 0.8-1.0] mg/L, $\mathrm{p}<0.0001)$, lower total protein (60.9 [IQR 57.5-65.0] vs 64.8 [60.9-68.3] g/L, $\mathrm{p}<0.0001)$. Besides, creatine kinase-MB (9.5 [IQR 7.3-13.4] vs 8.2 [IQR 6.6-10.2] IU/L, $\mathrm{p}<0.0001)$, lactate dehydrogenase (219.1 [IQR 180.9-273.6] vs 172.1 [IQR 150.4202.8] IU/L, p<0.0001), a-hydroxybutyrate dehydrogenase (181.6 [IQR 150.9-232.9] vs 140.6 [IQR 123.3-164.8] IU/L, p<0.0001), and hs-cTnl (0.02 [IQR 0.010.06 ] vs 0.01 [IQR $0.01-0.01] \mathrm{ng} / \mathrm{mL}, \mathrm{p}<0.0001$ ) were elevated in severe patients. PT (13.1 [IQR 12.4-14.0] vs 12.7 [IQR 12.1-13.4] s, p<0.0001), thrombin time (15.5 [IQR 14.7-16.4] vs 15.3 [IQR 14.5-16.0] s, p=0.0086), and fibrinogen (3.2 [IQR 2.8-3.7] vs 3.0 [IQR 2.6-3.3] g/L, p<0.0001) were elevated in severe patients. More severe patients suffered from liver injury, kidney injury, myocardial injury, and coagulation disorder. (Table 1)

Furthermore, all clinical adverse events were derived from severe cases and the length of hospitalization was longer in severe patients (16.0 [IQR 11.0-21.0) vs 14.0 [IQR 8.0-18.0] days, $\mathrm{p}<0.0001$ ) than in common cases. (Table 2)

\section{Risk factors for the incidence of severe patients}

In univariable regression, age, hypertension, diabetes, arrhythmia, malignant neoplasm, white blood cell count, lymphocyte percentage, hs-CRP, liver injury, acute kidney injury, cardiac injury as well as coagulation disorder were associated with the incidence of severe cases. (Table 3 ) The initial chest CT imaging and symptoms did not show significant correlations with severe cases of COVID-19 (Supplement table 1, 2). Importantly, results from multivariable analysis showed that age (OR: 1.02, 95\% confidence interval [95\% Cl]: 1.01-1.03, p=0.0003), arrhythmia (OR: 2.91, 95\% Cl: 1.45-5.86, p=0.0027), lymphocyte percentage (OR: 3.48, 95\% Cl: 2.58-4.69, p<0.0001), hs-CRP (OR: 3.73, 95\% Cl: 2.74-5.09, p<0.0001), and myocardial injury (OR: 3.02, 95\% Cl: 1.91-4.77, $\mathrm{p}<0.0001$ ) were independently associated with the incidence of severe cases. (Table 3 )

\section{Clinical outcomes in patients with or without myocardial injury}

After follow-up, the mortality rate was higher in patients with myocardial injury (16.2\%) than those without myocardial injury (0.3\%) (log-rank p value $<0.0001$, Figure 1A). What's more, the event-free survival rates (including the incidences of severe cases, ARDS and ICU admission) were similarly lower in patients with myocardial injury than those without myocardial injury (log-rank $p$ value $<0.0001$, Figure 1B-D). Furthermore, patients with myocardial injury showed significantly higher mortality rate, percentage of ARDS and ICU admission than those without myocardial injury irrespective of age (log-rank $\mathrm{p}$ value $<0.0001$, Figure $2 A, C, D$ ), whereas the incidence of severe cases seemed to be higher in patients with myocardial injury and age $\geq 75$ years old than those with myocardial injury and age $<75$ years old (Figure 2B). Moreover, the event-free survival rates (including mortality, the incidences of severe cases, ARDS and ICU admission) were even lower in patients with myocardial injury and arrhythmia than those with myocardial injury but without arrhythmia (log-rank $\mathrm{p}$ value $<0.0001$, Figure 3A-D). Interestingly, if myocardial injury co-existed with elevated hs-CRP, the clinical outcomes [mortality (Figure 4A), the incidences of severe cases (Figure 4B), ARDS (Figure 4C) and ICU admission (Figure 4D)] were extremely poor. Similar outcomes were found in patients with myocardial injury co-existed with abnormal lymphocyte percentage (log-rank p value $<0.0001$, Figure $5 A-D)$.

\section{Discussion}

Due to limited evidences, government guidance and clinical managements made the decisions based on the knowledge of risk factors for other similar infectious diseases such as influenza, Severe Acute Respiratory Syndromes (SARS), Middle East Respiratory Syndrome (MERS) to protect the millions of people at higher risk just according to age or serious comorbidities [17]. In this retrospective study, we found that severe COVID-19 cases were linked to poor clinical outcomes, such as respiratory failure, ARDS, ICU admission or even death. Several risk factors were identified to be associated with the incidence and progression of severe cases. Particularly, age, arrhythmia, myocardial injury, abnormalities of hs-CRP, and lymphocyte percentage were the independent risk factors for the incidence of severe cases. Of note, the presence of myocardial injury was associated with poor clinical outcomes including the progression of severe cases, which was more pronounced when co-existing with other factors. (Take-home figure)

Among COVID-19 patients, a minority of them experienced an abnormal clinical course which might lead to fatal outcomes. In this study, it was not surprising that all the deaths were derived from severe cases, the mortality was about 7\%, which was much higher than the reported overall mortality in COVID-19 patients, although the incidence of severe cases was similar with the results from meta-analysis [7]. Not only that, the length of hospital stay was longer in severe cases than those in common cases. These findings suggested severe cases might be associated with higher mortality and occupancy rate of medical sources. Consistent with previously described [10], more severe patients were elders; more patients had preexisting diseases, such as hypertention, diabetes, arrhythmia and so on; levels of inflammation was higher; multiple organs injury were more common, such as myocardial injury, kidney injury, immune injury, liver injury, coagulation disorders and so on. Consistently, previous study had reported the similar clinical characteristics in the deaths caused by COVID-19 [18], suggesting both severe cases and deceased cases might share a common pathophysiological basis. Learning from severe cases might favour the global success in fighting against COVID-19.

In this study, among the numerous factors, age, arrhythmia, myocardial injury, abnormalities of hs-CRP, and lymphocyte percentage were the independent risk factors for the incidence of severe cases. Consistently, it had been also reported that age $\geq 65$ years, preexisting cardiovascular or cerebrovascular 
diseases, lymphopenia, and elevation of hs-cTnl were associated with poor outcomes of COVID-19 pneumonia patients [19-21]. Moreover, patients with distinct inherited arrhythmia syndromes showed increased risk of COVID-19 [22]. According to previous studies, older age has been proved to be associated with mortality in SARS, MERS as well as COVID-19 [23], which might be attributed to the age-dependent defects in immunity and the excess production of inflammatory cytokines in elders. These defects might result in a failure in inhibition of viral replication and aggravated pro-inflammatory responses, which may lead to poor clinical outcomes [24]. Moreover, we also found that arrhythmia was independently associated with the severe cases. According to the "Diagnosis and Treatment of Novel Coronavirus Pneumonia (Trial Version 7)", elderly patients with preexisting diseases were more likely to be infected with SARS-CoV-2 and prone to be severe patients, especially those with hypertension, coronary heart disease, and diabetes. In our study, severe cases had higher levels of hs-CRP, which was also an independent risk factor. Similarly, previous evidence had showed that ICU patients with COVID-19 had higher plasma levels of inflammatory cytokines, including interleukin (IL)-2, IL-7, IL-10, tumor necrosis factor a and so on [2], suggesting that inflammatory cytokine storm syndrome due to uncontrolled and dysfunctional immune responses might contribute to the incidence and progression of severe cases.

In our study, we found that patients with myocardial injury had an increasing risk of mortality, higher incidence of severe cases, ARDS, and ICU admission. Consistently, previous studies showed that myocardial injury was associated with the fatal outcomes of COVID-19 patients [6]. Indeed, except myocardial injury, SARS-CoV-2 infection could lead to multiple organs injury, including lung, liver, kidney, as well as coagulation system [8]. However, only myocardial injury was independently associated with the incidence of severe cases. Moreover, myocardial injury was linked with the fast progression and poor clinical outcomes of severe cases, especially co-existing with old age, arrhythmia, or abnormalities of hs-CRP and lymphocyte percentage. These results suggested a critical impact of myocardial injury on the incidence and progression of severe cases.

Until now, the mechanisms of myocardial injury in COVID-19 patients were unclear and might be complex. However, it had been reported that patients with preexisting cardiovascular diseases were susceptible to suffer from myocardial injury during the clinical course of COVID-19 [14]. For these high risk patients, SARS-CoV-2 infection might further aggravate myocardial injury by the direct role of the virus, pro-inflammatory responses, hypoxia and so on. Evidence had showed that the SARS-CoV genome was positively detected in the heart, suggesting a direct viral myocardial infection [25]. Moreover, recent studies had shown that angiotensin-converting enzyme 2 (ACE2), which was highly expressed in heart, had a strong affinity to bind the Spike protein of SARS-CoV-2 [26]. Thus, we hypothesized that the myocardial injury in COVID-19 patients might be mediated by ACE2. Besides, recent evidence showed that plasma levels of troponin T were linearly correlated with the levels of hs-CRP, the activation or enhanced release of the inflammatory cytokines might lead to apoptosis or necrosis of myocardial cells, which indicated the contributions of pro-inflammatory responses [14]. Nevertheless, due to the limited evidence, this topic required further investigations.

Current knowledge of risk factors for severe COVID-19 mainly include age and comorbidities, which were unmodifiable. Thus, for elders or patients with some comorbidities, especially cardiovascular diseases, prevention seemed to be extremely important, avoiding infection should be specially recommended [27]. Based on the present findings, cardiac and immune injury might be the critical aspects for monitoring and interventions. Rather than encouraging avoidance of troponin testing, we recommended that hs-cTnl should be routinely detected and closely monitored in COVID-19 patients [28]. Under the basic clinical managements, protective strategy for heart and immune system should be emphasized to control the clinical severity and improve the clinical outcomes. For elders or patients with cardiovascular diseases, if COVID-19 was confirmed, cardioprotection should be taken immediately.

Our present study is a large-scale retrospective study to explore the risk factors involved in the incidence and prognosis of severe COVID-19 patients. The data were uniformly collected and the diagnosis and treatments were homogeneously performed. However, some limitations exist in our study. Firstly, because of the outbreak of COVID-19 in Wuhan, some data such as echocardiography data, electrocardiography data as well as arterial blood gas analysis were not collected in all patients due to the insufficiency of medical resources. Secondly, this is a single-centered study, further studies from multiple centers are needed.

\section{Conclusions}

For the first time, we identified that age, arrhythmia, myocardial injury, abnormalities of hs-CRP, and lymphocyte percentage were the independent risk factors for the incidence of severe COVID-19 cases. The presence of myocardial injury and its co-existing with other factors were greatly associated with poor clinical outcomes. Since no specific treatments could be applied to COVID-19, our findings may be beneficial to clinical decision-making and managements in order to improve clinical outcomes.

\section{Declarations}

\section{Acknowledgements}

We thank all the patients involved in the study.

\section{Authors' contributions}

HT, XD, QL, MH, LZ, and PL collected the epidemiological and clinical data. CL, JY, YYQ, YS, RC, XG, JK, FY and CH analyzed the data. HT, CL, JY and LH summarized all the data. HT, CL and JY drafted the manuscript. LH revised the final manuscript. All authors read and approved the final manuscript.

\section{Funding}

This work was supported by Scientific Research Project of Huoshenshan Hospital. 
The data used to support the findings of this study are available from the corresponding author upon request.

Ethics approval and consent to participate

This study was approved by the Human Ethics Committee, Huoshenshan Hospital (NO. HSSLL023) and conformed to the Declaration of Helsinki, oral consent was obtained from each patient when enrollment due to the rapid emergence of COVID-19.

Consent for publication

Not applicable.

Competing interests

The authors declare that they have no competing interests.

\section{References}

1. Chan JF, Yuan S, Kok KH, To KK, Chu H, Yang J, Xing F, Liu J, Yip CC, Poon RW et al: A familial cluster of pneumonia associated with the 2019 novel coronavirus indicating person-to-person transmission: a study of a family cluster. Lancet 2020, 395(10223):514-523.

2. Huang C, Wang Y, Li X, Ren L, Zhao J, Hu Y, Zhang L, Fan G, Xu J, Gu X et al: Clinical features of patients infected with 2019 novel coronavirus in Wuhan, China. Lancet 2020, 395(10223):497-506.

3. Chen N, Zhou M, Dong X, Qu J, Gong F, Han Y, Qiu Y, Wang J, Liu Y, Wei Y et al: Epidemiological and clinical characteristics of 99 cases of 2019 novel coronavirus pneumonia in Wuhan, China: a descriptive study. Lancet 2020, 395(10223):507-513.

4. Guan WJ, Ni ZY, Hu Y, Liang WH, Ou CQ, He JX, Liu L, Shan H, Lei CL, Hui DSC et al: Clinical Characteristics of Coronavirus Disease 2019 in China. N Engl J Med 2020.

5. Wang D, Hu B, Hu C, Zhu F, Liu X, Zhang J, Wang B, Xiang H, Cheng Z, Xiong Y et al: Clinical Characteristics of 138 Hospitalized Patients With 2019 Novel Coronavirus-Infected Pneumonia in Wuhan, China. JAMA 2020.

6. Shi S, Qin M, Shen B, Cai Y, Liu T, Yang F, Gong W, Liu X, Liang J, Zhao Q et al: Association of Cardiac Injury With Mortality in Hospitalized Patients With COVID-19 in Wuhan, China. JAMA Cardiol 2020.

7. Sun P, Qie S, Liu Z, Ren J, Li K, Xi J: Clinical characteristics of hospitalized patients with SARS-CoV-2 infection: A single arm meta-analysis. J Med Virol 2020.

8. Zhou F, Yu T, Du R, Fan G, Liu Y, Liu Z, Xiang J, Wang Y, Song B, Gu X et al: Clinical course and risk factors for mortality of adult inpatients with CoVID19 in Wuhan, China: a retrospective cohort study. Lancet 2020, 395(10229):1054-1062.

9. Yang X, Yu Y, Xu J, Shu H, Xia J, Liu H, Wu Y, Zhang L, Yu Z, Fang M et al: Clinical course and outcomes of critically ill patients with SARS-CoV-2 pneumonia in Wuhan, China: a single-centered, retrospective, observational study. Lancet Respir Med 2020.

10. Feng Y, Ling Y, Bai T, Xie Y, Huang J, Li J, Xiong W, Yang D, Chen R, Lu F et al: CoVID-19 with Different Severity: A Multi-center Study of Clinical Features. Am J Respir Crit Care Med 2020.

11. World Health Organization: Clinical management of severe acute respiratory infection when Novel coronavirus (nCoV) infection is suspected: interim guidance. Jan 11, 2020

https://www.who.int/internalpublicationsdetail/clinicalmanagementofsevereacuterespiratoryinfectionwhennovelcoronavirus(ncov)infectionissuspected (accessed Jan 20, 2020).

12. National Health Commission of People's Republic of China: Chinese management guideline for COVID-19 (version 5.0). Feb 8, 2020

http://www.nhc.gov.cn/yzygj/s7653p/202002/d4b895337e19445f8d728fcaf1e3e13a/files/ab6bec7f93e64e7f998d802991203cd6.pdf (accessed Feb 8, 2020; in Chinese).

13. Ranieri VM, Rubenfeld GD, Thompson BT, Ferguson ND, Caldwell E, Fan E, Camporota L, Slutsky AS: Acute respiratory distress syndrome: the Berlin Definition. JAMA 2012, 307(23):2526-2533.

14. Guo T, Fan Y, Chen M, Wu X, Zhang L, He T, Wang H, Wan J, Wang X, Lu Z: Cardiovascular Implications of Fatal Outcomes of Patients With Coronavirus Disease 2019 (COVID-19). JAMA Cardiol 2020.

15. Jha V, Arici M, Collins AJ, Garcia-Garcia G, Hemmelgarn BR, Jafar TH, Pecoits-Filho R, Sola L, Swanepoel CR, Tchokhonelidze I et al: Understanding kidney care needs and implementation strategies in low- and middle-income countries: conclusions from a "Kidney Disease: Improving Global Outcomes" (KDIGO) Controversies Conference. Kidney Int 2016, 90(6):1164-1174.

16. Kwo PY, Cohen SM, Lim JK: ACG Clinical Guideline: Evaluation of Abnormal Liver Chemistries. Am J Gastroentero/2017, 112(1):18-35.

17. Jordan RE, Adab P, Cheng KK: Covid-19: risk factors for severe disease and death. BMJ 2020, 368:m1198.

18. Chen T, Wu D, Chen H, Yan W, Yang D, Chen G, Ma K, Xu D, Yu H, Wang H et al: Clinical characteristics of 113 deceased patients with coronavirus disease 2019: retrospective study. BMJ 2020, 368:m1091.

19. Du RH, Liang LR, Yang CQ, Wang W, Cao TZ, Li M, Guo GY, Du J, Zheng CL, Zhu Q et al: Predictors of Mortality for Patients with CoVID-19 Pneumonia Caused by SARS-CoV-2: A Prospective Cohort Study. Eur Respir J 2020.

20. Chen G, Wu D, Guo W, Cao Y, Huang D, Wang H, Wang T, Zhang X, Chen H, Yu H et al: Clinical and immunological features of severe and moderate coronavirus disease 2019. J Clin Invest 2020. 
21. Guan WJ, Liang WH, Zhao Y, Liang HR, Chen ZS, Li YM, Liu XQ, Chen RC, Tang CL, Wang T et al: Comorbidity and its impact on 1590 patients with Covid-19 in China: A Nationwide Analysis. Eur Respir J 2020.

22. Wu Cl, Postema PG, Arbelo E, Behr ER, Bezzina CR, Napolitano C, Robyns T, Probst V, Schulze-Bahr E, Remme CA et al: SARS-CoV-2, CoVID-19 and inherited arrhythmia syndromes. Heart Rhythm 2020.

23. Choi KW, Chau TN, Tsang O, Tso E, Chiu MC, Tong WL, Lee PO, Ng TK, Ng WF, Lee KC et al: Outcomes and prognostic factors in 267 patients with severe acute respiratory syndrome in Hong Kong. Ann Intern Med 2003, 139(9):715-723.

24. Opal SM, Girard TD, Ely EW: The immunopathogenesis of sepsis in elderly patients. Clin Infect Dis 2005, 41 Suppl 7:S504-512.

25. Oudit GY, Kassiri Z, Jiang C, Liu PP, Poutanen SM, Penninger JM, Butany J: SARS-coronavirus modulation of myocardial ACE2 expression and inflammation in patients with SARS. Eur J Clin Invest 2009, 39(7):618-625.

26. Zou X, Chen K, Zou J, Han P, Hao J, Han Z: Single-cell RNA-seq data analysis on the receptor ACE2 expression reveals the potential risk of different human organs vulnerable to 2019-nCoV infection. Front Med 2020.

27. Clerkin KJ, Fried JA, Raikhelkar J, Sayer G, Griffin JM, Masoumi A, Jain SS, Burkhoff D, Kumaraiah D, Rabbani L et al: Coronavirus Disease 2019 (CoVID19) and Cardiovascular Disease. Circulation 2020.

28. Chapman AR, Bularga A, Mills NL: High-Sensitivity Cardiac Troponin Can Be An Ally in the Fight Against COVID-19. Circulation 2020.

\section{Tables}

Table 1: Demographic information, clinical features, chest CT imaging and laboratory data. 


\begin{tabular}{|c|c|c|c|c|}
\hline & $\begin{array}{l}\text { All patients } \\
(n=1646)\end{array}$ & $\begin{array}{l}\text { Common patients } \\
(n=1319)\end{array}$ & $\begin{array}{l}\text { Severe patients } \\
(n=327)\end{array}$ & $p$ value \\
\hline \multicolumn{5}{|l|}{ Demographics } \\
\hline Age, years & $60.0(50.0-68.0)$ & $58.0(48.0-67.0)$ & $65.0(56.0-72.0)$ & $<0.0001$ \\
\hline Males, n (\%) & $830(50.4 \%)$ & $666(50.5 \%)$ & $164(50.2 \%)$ & 0.91 \\
\hline Current smoker, n (\%) & $93(5.7 \%)$ & $76(5.8 \%)$ & $17(5.2 \%)$ & 0.69 \\
\hline Current drinker, $\mathrm{n}(\%)$ & $56(3.4 \%)$ & $50(3.8 \%)$ & $6(1.8 \%)$ & 0.08 \\
\hline \multicolumn{5}{|l|}{ Initial symptoms } \\
\hline Fever $\left(\geqq 37.3^{\circ} \mathrm{C}\right), \mathrm{n}(\%)$ & $1034(62.8 \%)$ & $850(64.4 \%)$ & $184(56.3 \%)$ & 0.0062 \\
\hline Cough/sputum, n (\%) & $277(16.8 \%)$ & $216(16.4 \%)$ & $61(18.7 \%)$ & 0.32 \\
\hline Short of breath, n (\%) & $71(4.3 \%)$ & $47(3.6 \%)$ & $24(7.3 \%)$ & 0.0026 \\
\hline Fatigue, n (\%) & $108(6.6 \%)$ & $81(6.1 \%)$ & $27(8.3 \%)$ & 0.17 \\
\hline Myalgia, n (\%) & $22(1.3 \%)$ & $18(1.4 \%)$ & $4(1.2 \%)$ & 1.0 \\
\hline Nausea/vomiting, n (\%) & $9(0.6 \%)$ & $7(0.5 \%)$ & $2(0.6 \%)$ & 1.0 \\
\hline Diarrhea, n (\%) & $26(1.6 \%)$ & $17(1.3 \%)$ & $9(2.8 \%)$ & 0.057 \\
\hline Stuffy/runny noses, n (\%) & $10(0.6 \%)$ & $8(0.6 \%)$ & $2(0.6 \%)$ & 1.0 \\
\hline Throat discomfort, n (\%) & $24(1.5 \%)$ & $20(1.5 \%)$ & $4(1.2 \%)$ & 0.89 \\
\hline Dizziness/headache, n (\%) & $17(1.0 \%)$ & $15(1.1 \%)$ & $2(0.6 \%)$ & 0.59 \\
\hline No symptoms, n (\%) & $48(2.9 \%)$ & $40(3.0 \%)$ & $8(2.4 \%)$ & 0.57 \\
\hline \multicolumn{5}{|l|}{ Comorbidities } \\
\hline Hypertension, n (\%) & $490(29.8 \%)$ & $352(26.7 \%)$ & $138(42.2 \%)$ & $<0.0001$ \\
\hline Diabetes, n (\%) & $220(13.4 \%)$ & $154(11.7 \%)$ & $66(20.2 \%)$ & $<0.0001$ \\
\hline Arrhythmia, n (\%) & $53(3.2 \%)$ & $28(2.1 \%)$ & $25(7.6 \%)$ & $<0.0001$ \\
\hline Malignant neoplasm, n (\%) & $28(1.7 \%)$ & $18(1.4 \%)$ & $10(3.1 \%)$ & 0.034 \\
\hline Hyperlipemia, n (\%) & $25(1.5 \%)$ & $20(1.5 \%)$ & $5(1.5 \%)$ & 0.99 \\
\hline \multicolumn{5}{|l|}{ Chest CT imaging } \\
\hline Unilateral, n (\%) & $133(8.1 \%)$ & $105(8.0 \%)$ & $28(8.6 \%)$ & 0.72 \\
\hline Bilateral, n (\%) & $1312(79.7 \%)$ & $1056(80.1 \%)$ & $256(78.3 \%)$ & 0.48 \\
\hline Ground-glass opacity, n (\%) & $1482(90.0 \%)$ & 1191 (90.3\%) & $291(89.0 \%)$ & 0.48 \\
\hline \multicolumn{5}{|l|}{ Blood routine test } \\
\hline Leucocytes, $\times 10^{9} / \mathrm{L}$ & $5.8(4.8-7.1)$ & $5.7(4.7-6.8)$ & $6.7(5.3-8.3)$ & $<0.0001$ \\
\hline Neutrophils, $\times 10^{9} / \mathrm{L}$ & $3.6(2.7-4.7)$ & $3.4(2.7-4.4)$ & $4.6(3.4-6.4)$ & $<0.0001$ \\
\hline Neutrophil percentage, \% & $62.2(56.0-68.7)$ & $60.7(54.8-66.5)$ & $69.9(62.6-78.0)$ & $<0.0001$ \\
\hline Lymphocyte, $\times 10^{9} / \mathrm{L}$ & $1.5(1.2-1.9)$ & $1.6(1.3-1.9)$ & $1.2(0.8-1.5)$ & $<0.0001$ \\
\hline Lymphocyte percentage, \% & $26.8(21.1-32.7)$ & $28.2(23.0-33.6)$ & $18.9(12.9-26.1)$ & $<0.0001$ \\
\hline Eosnophil, $\times 10^{9} / \mathrm{L}$ & $0.11(0.07-0.17)$ & $0.11(0.07-0.18)$ & $0.10(0.06-0.17)$ & 0.0068 \\
\hline Eosnophil percentage, \% & $1.9(1.2-3.0)$ & $2.0(1.3-3.1)$ & $1.6(0.9-2.6)$ & $<0.0001$ \\
\hline Basophil, $\times 10^{9} / \mathrm{L}$ & $0.02(0.01-0.03)$ & $0.02(0.02-0.03)$ & $0.02(0.01-0.03)$ & $<0.0001$ \\
\hline Basophil percentage, \% & $0.37(0.23-0.50)$ & $0.40(0.28-0.50)$ & $0.28(0.15-0.40)$ & $<0.0001$ \\
\hline Monocyte, $\times 10^{9} / \mathrm{L}$ & $0.44(0.36-0.55)$ & $0.43(0.35-0.54)$ & $0.47(0.37-0.58)$ & 0.0018 \\
\hline Monocyte percentage, \% & $7.7(6.5-9.0)$ & $7.7(6.6-9.0)$ & $7.6(6.0-8.9)$ & 0.010 \\
\hline Hemoglobin, g/L & $124.0(114.0-134.5)$ & $125.5(116.0-136.0)$ & $116.3(104.5-127.7)$ & $<0.0001$ \\
\hline
\end{tabular}




\begin{tabular}{|c|c|c|c|c|}
\hline Platelets, $\times 10^{9} / \mathrm{L}$ & $231.0(188.5-278.2)$ & $231.0(191.0-277.0)$ & $228.0(172.3-287.5)$ & 0.10 \\
\hline \multicolumn{5}{|l|}{ Inflammatory biomarkers } \\
\hline hs-CRP, mg/L & $2.7(1.0-10.3)$ & $2.0(0.8-5.9)$ & $15.6(4.5-45.0)$ & $<0.0001$ \\
\hline \multicolumn{5}{|l|}{ Liver function } \\
\hline ALT, IU/L & $24.7(15.4-40.8)$ & $23.9(15.3-38.9)$ & $29.3(17.6-50.4)$ & $<0.0001$ \\
\hline Aspartate aminotransferase, IU/L & $20.2(16.0-27.1)$ & $19.4(15.6-25.7)$ & $22.4(18.3-32.5)$ & $<0.0001$ \\
\hline Total protein, g/L & $64.2(60.2-67.8)$ & $64.8(60.9-68.3)$ & $60.9(57.5-65.0)$ & $<0.0001$ \\
\hline Globulin, g/L & $27.0(24.7-29.7)$ & $26.9(24.6-29.6)$ & $27.4(24.7-30.1)$ & 0.29 \\
\hline Total bilirubin, $\mu \mathrm{mol} / \mathrm{L}$ & $9.1(7.2-11.7)$ & $9.0(7.2-11.5)$ & $9.5(7.2-12.4)$ & 0.044 \\
\hline Y-glutamyl transpeptidase, IU/L & $31.4(20.6-51.3)$ & $30.2(20.1-48.4)$ & $37.0(23.1-60.4)$ & $<0.0001$ \\
\hline Liver injury, n (\%) & $294(17.9 \%)$ & $212(16.1 \%)$ & $82(25.1 \%)$ & $<0.0001$ \\
\hline \multicolumn{5}{|l|}{ Renal function } \\
\hline Urea nitrogen, $\mathrm{mmol} / \mathrm{L}$ & $4.4(3.6-5.4)$ & $4.3(3.6-5.2)$ & $5.0(4.0-6.5)$ & $<0.0001$ \\
\hline Creatinine, $\mu \mathrm{mol} / \mathrm{L}$ & $63.8(54.5-74.9)$ & $63.8(54.3-74.3)$ & $63.9(55.3-78.3)$ & 0.12 \\
\hline Cystatin C, mg/L & $0.9(0.8-1.1)$ & $0.9(0.8-1.0)$ & $1.0(0.9-1.2)$ & $<0.0001$ \\
\hline Acute kidney injury, n (\%) & $19(1.2 \%)$ & $5(0.4 \%)$ & $14(4.3 \%)$ & $<0.0001$ \\
\hline \multicolumn{5}{|l|}{ Cardiac biomarkers } \\
\hline Creatine kinase, IU/L & $44.7(32.4-66.5)$ & $45.4(33.3-66.2)$ & $41.2(29.3-69.6)$ & 0.073 \\
\hline Creatine kinase-MB, IU/L & $8.4(6.7-10.7)$ & $8.2(6.6-10.2)$ & $9.5(7.3-13.4)$ & $<0.0001$ \\
\hline Lactic dehydrogenase, IU/L & $178.9(154.4-213.5)$ & $172.1(150.4-202.8)$ & $219.1(180.9-273.6)$ & $<0.0001$ \\
\hline a-hydroxybutyrate dehydrogenase, IU/L & $146.7(125.6-177.5)$ & $140.6(123.3-164.8)$ & $181.6(150.9-232.9)$ & $<0.0001$ \\
\hline hs-cTnl, ng/mL & $0.01(0.01-0.02)$ & $0.01(0.01-0.01)$ & $0.02(0.01-0.06)$ & $<0.0001$ \\
\hline myocardial injury, n (\%) & $117(7.1 \%)$ & $50(3.8 \%)$ & $67(20.5 \%)$ & $<0.0001$ \\
\hline \multicolumn{5}{|l|}{ Coagulation profiles } \\
\hline PT, s & $12.7(12.2-13.5)$ & $12.7(12.1-13.4)$ & $13.1(12.4-14.0)$ & $<0.0001$ \\
\hline Activated partial thromboplastin time, s & $27.8(25.9-30.0)$ & $27.8(25.9-30.0)$ & $27.8(25.9-29.9)$ & 0.73 \\
\hline Thrombin time, s & $15.3(14.6-16.1)$ & $15.3(14.5-16.0)$ & $15.5(14.7-16.4)$ & 0.0086 \\
\hline Fibrinogen, $\mathrm{g} / \mathrm{L}$ & $3.0(2.6-3.4)$ & $3.0(2.6-3.3)$ & $3.2(2.8-3.7)$ & $<0.0001$ \\
\hline Coagulation disorder & $28(1.7 \%)$ & $15(1.1 \%)$ & $13(4.0 \%)$ & $<0.0001$ \\
\hline
\end{tabular}

Data were expressed as $\mathrm{n}(\%)$ and median (IQR). ALT=alanine aminotransferase. hs-CRP=hypersensitive $\mathrm{C}$ reactive protein. hs-cTnl=hypersensitive cardiac troponin I. PT=prothrombin time.

Table 2: Treatments and outcomes. 


\begin{tabular}{|c|c|c|c|c|}
\hline & $\begin{array}{l}\text { All patients } \\
(n=1646)\end{array}$ & $\begin{array}{l}\text { Common patients } \\
(n=1319)\end{array}$ & $\begin{array}{l}\text { Severe patients } \\
(n=327)\end{array}$ & $p$ value \\
\hline \multicolumn{5}{|l|}{ Treatments } \\
\hline Abidor hydrochloride, n (\%) & $751(45.6 \%)$ & $575(43.6 \%)$ & $176(53.8 \%)$ & 0.0009 \\
\hline Oseltamivir phosphate, $\mathrm{n}(\%)$ & $194(11.8 \%)$ & $155(11.8 \%)$ & $39(11.9 \%)$ & 0.93 \\
\hline Ribavirin, n (\%) & $77(4.7 \%)$ & $46(3.5 \%)$ & $31(9.5 \%)$ & $<0.0001$ \\
\hline Moxifloxacin hydrochloride, $\mathrm{n}(\%)$ & $493(30.0 \%)$ & $359(27.2 \%)$ & $134(41.0 \%)$ & $<0.0001$ \\
\hline Vitamin $\mathrm{C}, \mathrm{n}(\%)$ & $481(29.2 \%)$ & $369(28.0 \%)$ & $112(34.3 \%)$ & 0.026 \\
\hline Thymalfasin, $\mathrm{n}(\%)$ & $123(7.5 \%)$ & $73(5.5 \%)$ & $50(15.3 \%)$ & $<0.0001$ \\
\hline Recombinant interference, $\mathrm{n}(\%)$ & $104(6.3 \%)$ & $84(6.4 \%)$ & $20(6.1 \%)$ & 0.87 \\
\hline Methylprednisolone sodium succinate, n (\%) & $159(9.7 \%)$ & $80(6.1 \%)$ & $79(24.2 \%)$ & $<0.0001$ \\
\hline Prednisone acetate, n (\%) & $56(3.4 \%)$ & $38(2.9 \%)$ & $18(5.5 \%)$ & 0.019 \\
\hline \multicolumn{5}{|l|}{ Outcomes } \\
\hline Death, n (\%) & $23(1.4 \%)$ & $0(0.0 \%)$ & $23(7.0 \%)$ & $<0.0001$ \\
\hline Respiratory failure, n (\%) & $57(3.5 \%)$ & $0(0.0 \%)$ & $57(17.4 \%)$ & $<0.0001$ \\
\hline ARDS, n (\%) & $73(4.4 \%)$ & $0(0.0 \%)$ & $73(22.3 \%)$ & $<0.0001$ \\
\hline Mechanical ventilation, $\mathrm{n}(\%)$ & $43(2.6 \%)$ & $0(0.0 \%)$ & $43(13.2 \%)$ & $<0.0001$ \\
\hline ICU admission, $\mathrm{n}(\%)$ & $60(3.7 \%)$ & $0(0.0 \%)$ & $60(18.4 \%)$ & $<0.0001$ \\
\hline Hospital length of stay, days & $14.0(9.0-19.0)$ & $14.0(8.0-18.0)$ & $16.0(11.0-21.0)$ & $<0.0001$ \\
\hline Time to death, days & - & - & $26.0(17.0-31.0)$ & - \\
\hline Time to severe illness, days & - & - & $20.0(14.0-26.0)$ & - \\
\hline Time to respiratory failure, days & - & - & $18.5(14.0-25.0)$ & - \\
\hline Time to ARDS, days & - & - & $18.0(14.0-26.5)$ & - \\
\hline Time to mechanical ventilation, days & - & - & $20.0(13.0-26.0)$ & - \\
\hline Time to ICU admission, days & - & - & $18.0(14.0-25.0)$ & - \\
\hline ICU length of stay, days & - & - & $5.5(3.0-9.0)$ & - \\
\hline Time to normothermia, days & $24.0(17.0-32.0)$ & $23.0(17.0-32.0)$ & $26.0(17.0-33.0)$ & 0.35 \\
\hline Time to inflammatory resorption, days & $30.0(23.0-36.0)$ & $30.0(23.0-36.0)$ & $30.0(25.0-37.0)$ & 0.13 \\
\hline Time to viral shedding, days & $29.0(24.0-36.0)$ & $29.0(24.0-36.0)$ & $30.0(24.0-35.0)$ & 0.94 \\
\hline
\end{tabular}

Data were expressed as $\mathrm{n}(\%)$ and median (IQR). ARDS=acute respiratory distress syndrome. ICU=intensive care unit. IQR=interquartile range. Time=time from illness onset to clinical outcomes.

Table 3: Risk factors for the incidence of severe COVID-19 patients 


\begin{tabular}{|c|c|c|c|c|}
\hline \multirow[t]{2}{*}{ Variables } & \multirow{2}{*}{$\begin{array}{l}\text { Univariable } \\
\text { OR }(95 \% \mathrm{Cl})\end{array}$} & \multirow[t]{2}{*}{$p$ value } & Multivariable & \multirow[t]{2}{*}{$p$ value } \\
\hline & & & OR $(95 \% \mathrm{Cl})$ & \\
\hline \multicolumn{5}{|c|}{ Demographics and clinical characteristics } \\
\hline Age (per 1 year) & $1.04(1.03-1.05)$ & $<0.0001$ & $1.02(1.01-1.03)$ & 0.0003 \\
\hline Males (vs females) & $0.99(0.77-1.26)$ & 0.91 & & \\
\hline Current smoker (vs non-smoker) & $0.90(0.52-1.54)$ & 0.69 & & \\
\hline \multicolumn{5}{|l|}{ Comorbidities (vs not) } \\
\hline Hypertension & $2.02(1.56-2.58)$ & $<0.0001$ & & \\
\hline Diabetes & $1.91(1.39-2.63)$ & $<0.0001$ & & \\
\hline Arrhythmia & $3.82(2.19-6.64)$ & $<0.0001$ & $2.91(1.45-5.86)$ & 0.0027 \\
\hline Malignant neoplasm & $2.28(1.04-4.99)$ & 0.039 & & \\
\hline Hyperlipemia & $1.01(0.38-2.71)$ & 0.99 & & \\
\hline \multicolumn{5}{|l|}{ Laboratory findings } \\
\hline \multicolumn{5}{|l|}{ White blood cell count, $\times 10^{9} / \mathrm{L}$} \\
\hline $3.5-9.5$ & 1 (ref) & & & \\
\hline$<3.5$ or $>9.5$ & $3.23(2.29-4.56)$ & $<0.0001$ & & \\
\hline \multicolumn{5}{|l|}{ Lymphocyte percentage, $\%$} \\
\hline $2-50 \%$ & 1 (ref) & & 1 (ref) & \\
\hline$<20$ or $>50 \%$ & $6.93(5.31-9.04)$ & $<0.0001$ & $3.48(2.58-4.69)$ & $<0.0001$ \\
\hline \multicolumn{5}{|l|}{ hs-CRP, mg/L } \\
\hline 4 & 1 (ref) & & 1 (ref) & \\
\hline$>4$ & $6.76(5.10-8.95)$ & $<0.0001$ & $3.73(2.74-5.09)$ & $<0.0001$ \\
\hline Liver injury ( $v s$ not) & $1.75(1.31-2.34)$ & 0.0002 & & \\
\hline Acute kidney injury (vs not) & $11.76(4.20-32.88)$ & $<0.0001$ & & \\
\hline Cardiac injury (vs not) & $6.54(4.43-9.66)$ & $<0.0001$ & $3.02(1.91-4.77)$ & $<0.0001$ \\
\hline Coagulation disorder (vs not) & $3.60(1.70-7.64)$ & 0.0009 & & \\
\hline
\end{tabular}

$95 \% \mathrm{Cl}=95 \%$ confidence interval. OR=odds ratio. Abbreviations are as table 1.

\section{Figures}


A

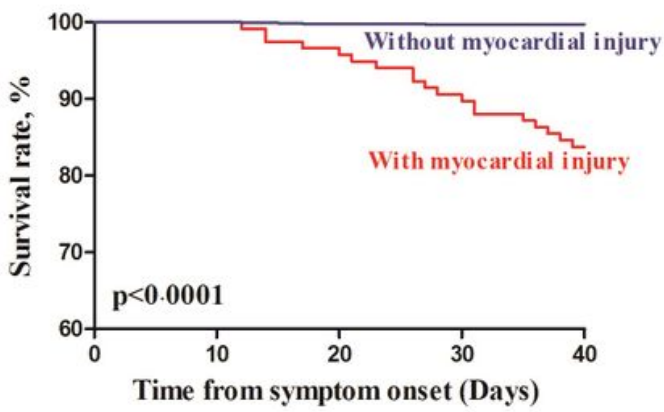

No. at risk

$\begin{array}{lrrrrr}\text { With myocardial injury } & 117 & 117 & 112 & 105 & 98 \\ \text { Without myocardial injury } & 1529 & 1529 & 1526 & 1525 & 1525\end{array}$

C

No. at risk

With myocardial injury $\quad 117$

Without myocardial injury 1529

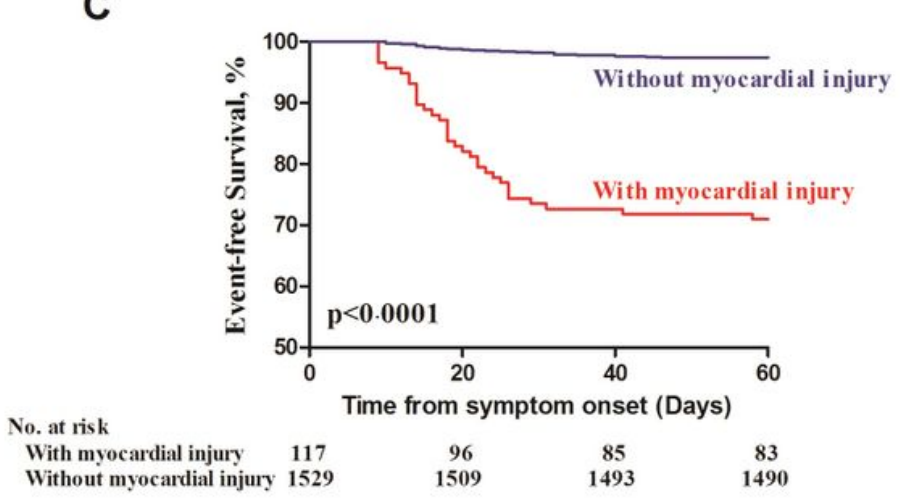

B

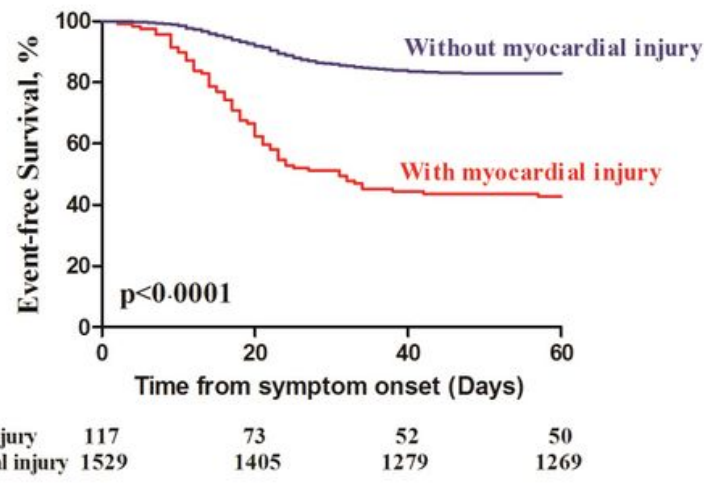

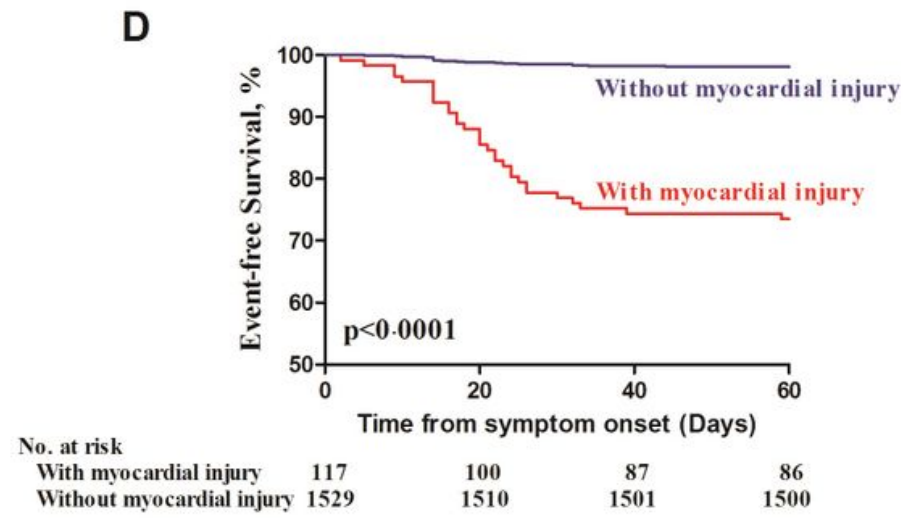

\section{Figure 1}

Event-free survival in COVID-19 patients according to myocardial injury. Kaplan-Meier plot of survival rate (A), event-free (the incidence of severe cases) survival (B), event-free (the incidence of ARDS) survival (C), and event-free (ICU admission) survival (D) during the time from symptom onset in patients with myocardial injury (red) and without myocardial injury (blue). ARDS=acute respiratory distress syndrome. COVID-19=coronavirus disease 2019. ICU=intensive care unit. 

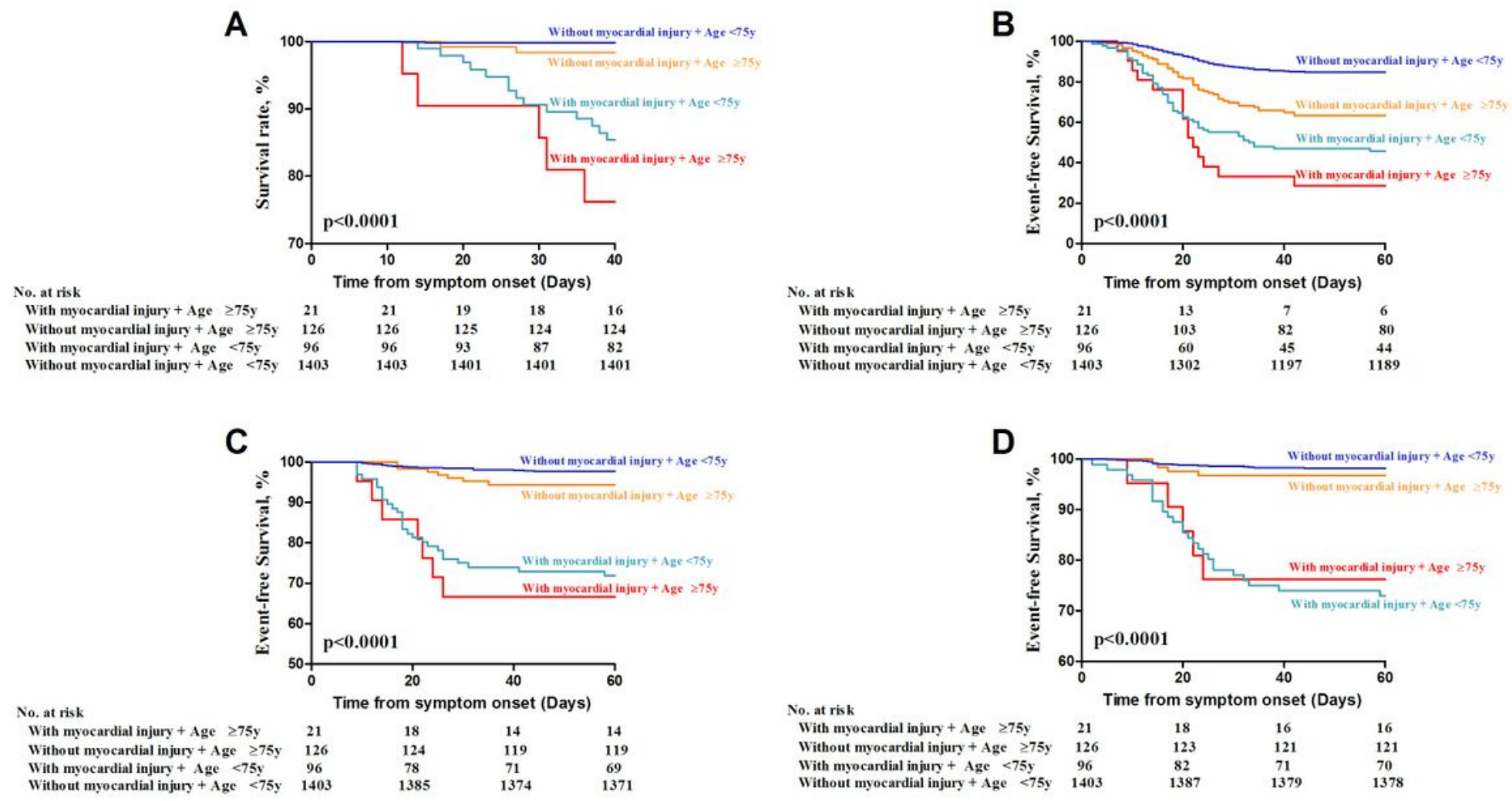

Figure 2

Event-free survival in COVID-19 patients according to myocardial injury and age. Kaplan-Meier plot of survival rate (A), event-free (the incidence of severe cases) survival (B), event-free (the incidence of ARDS) survival (C), and event-free (ICU admission) survival (D) among patients in age $\geq 75$ years old or age $<75$ years old with or without myocardial injury.
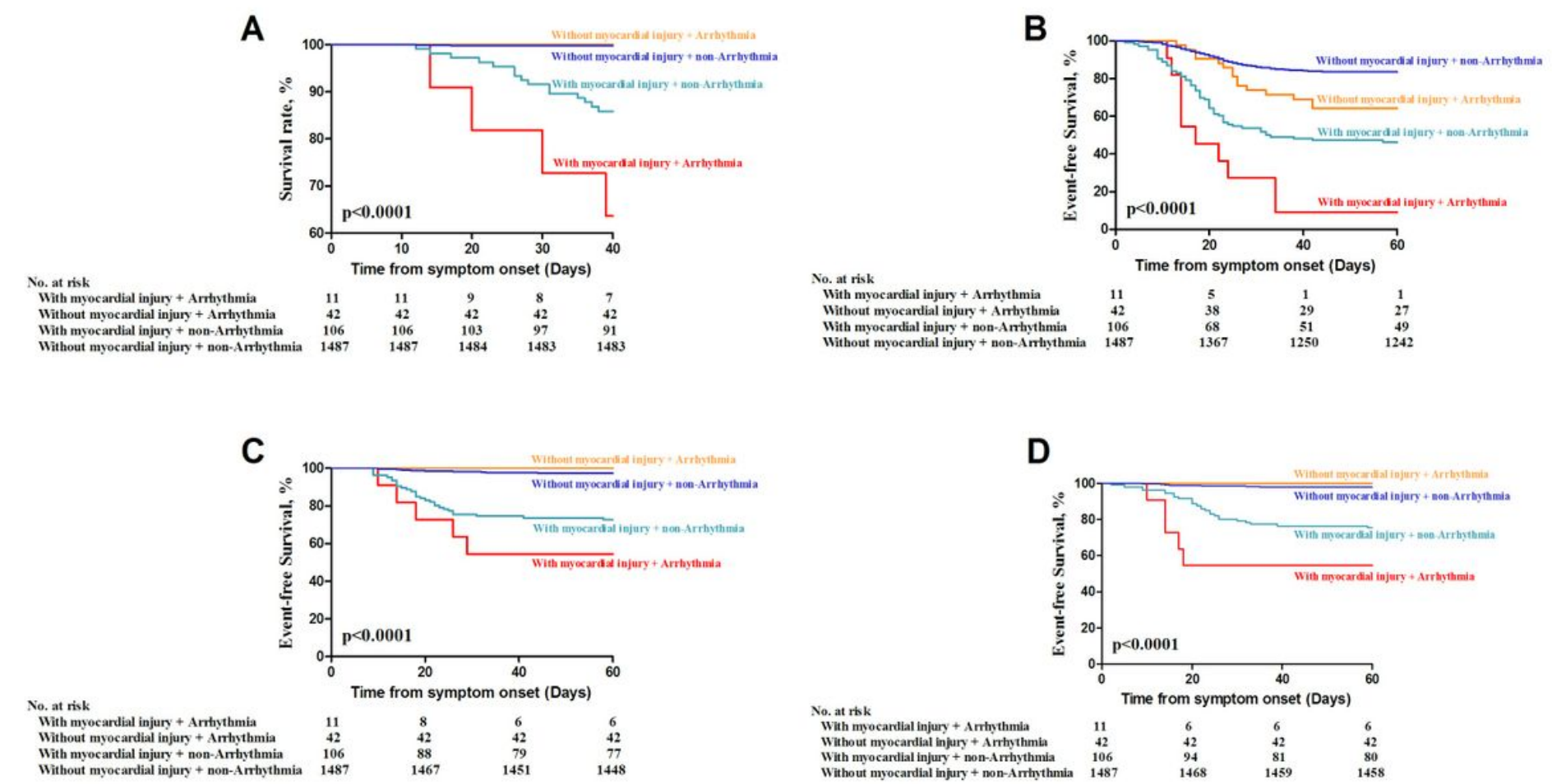

Figure 3 
Event-free survival in COVID-19 patients according to myocardial injury and arrhythmia. Kaplan-Meier plot of survival rate (A), event-free (the incidence of severe cases) survival (B), event-free (the incidence of ARDS) survival (C), and event-free (ICU admission) survival (D) among patients with or without myocardial injury co-existed with or without arrhythmia.

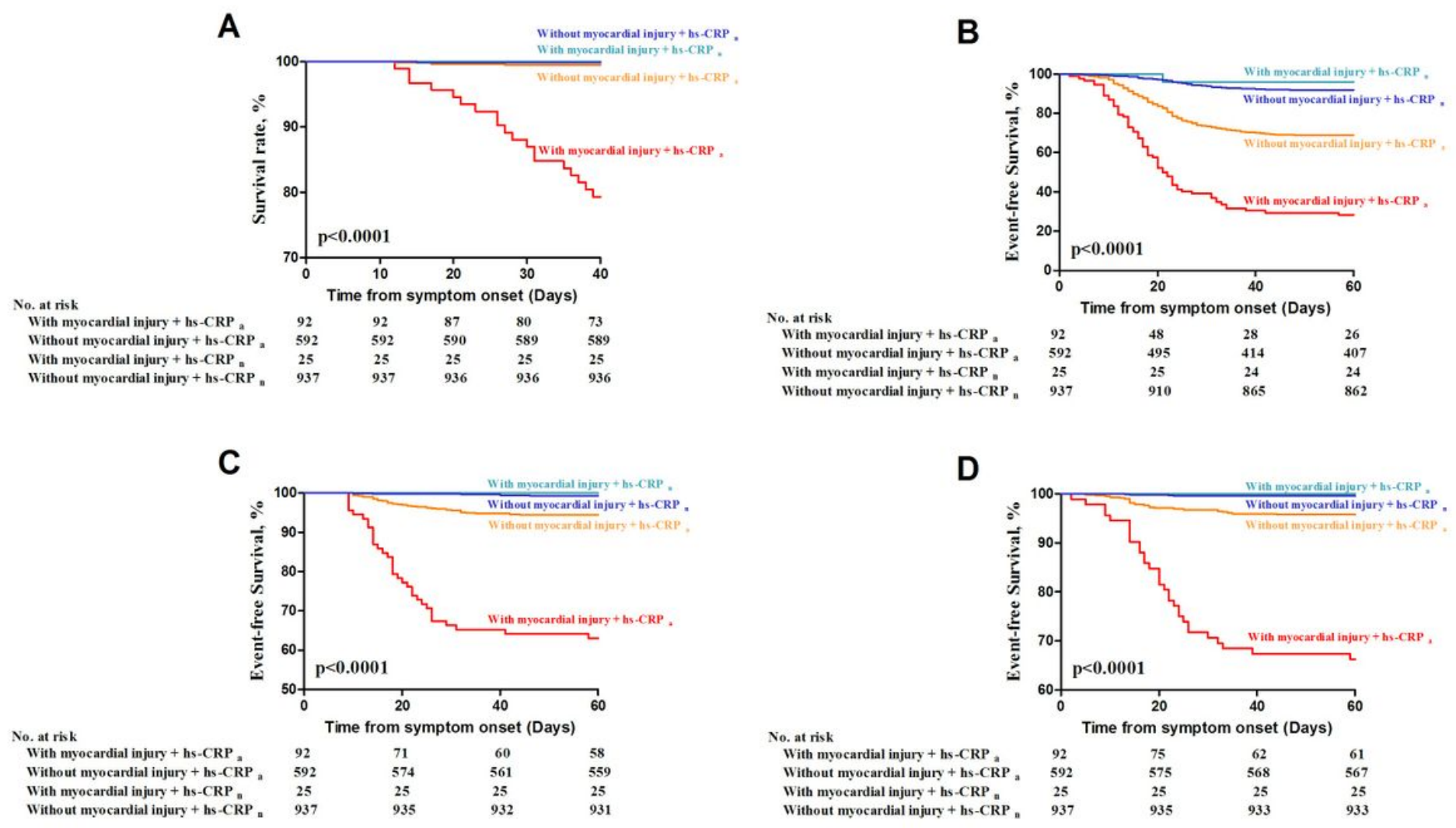

Figure 4

Event-free survival in COVID-19 patients according to myocardial injury and hs-CRP. Kaplan-Meier plot of survival rate (A), event-free (the incidence of severe cases) survival (B), event-free (the incidence of ARDS) survival (C), and event-free (ICU admission) survival (D) among patients with or without myocardial injury co-existed with hs-CRPa or hs-CRPn. hs-CRPa=abnormal hypersensitive $\mathrm{C}$ reactive protein $(>4 \mathrm{mg} / \mathrm{L})$. hs-CRPn=normal hypersensitive $\mathrm{C}$ reactive protein $(\leq 4 \mathrm{mg} / \mathrm{L})$. 
A

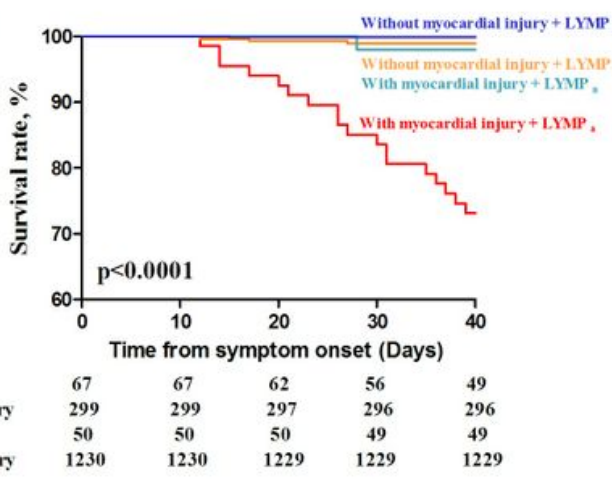

No. at risk

LYMP $_{\mathrm{a}}$ With myocardial injury

LYMP $_{\text {a }}$ Without myocardial inju

LYMP, With myocardial injury

LYMP $_{\mathrm{n}}$ Without myocardial injury
C

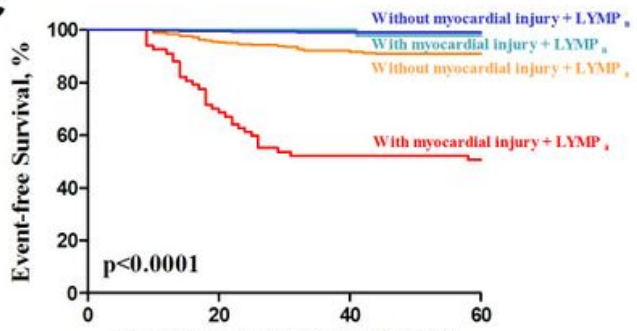

No. at risk

LYMPP $_{\mathrm{a}}$ With nyocardial injury

LYMP a Without nvocardial injury

LYMP $_{n}$ With myocardial injury

LYMPP $_{n}$ Without myocardial injury
Time from symptom onset (Days)

$\begin{array}{cccc}67 & 46 & 35 & 34 \\ 299 & 285 & 274 & 272 \\ 50 & 50 & 50 & 49 \\ 1230 & 1224 & 1219 & 1218\end{array}$

B

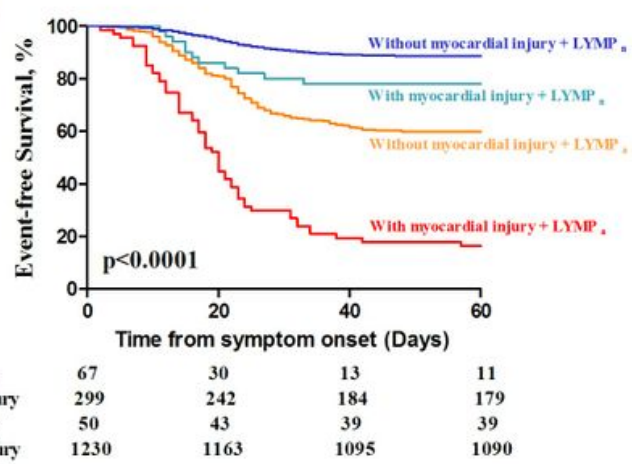

D

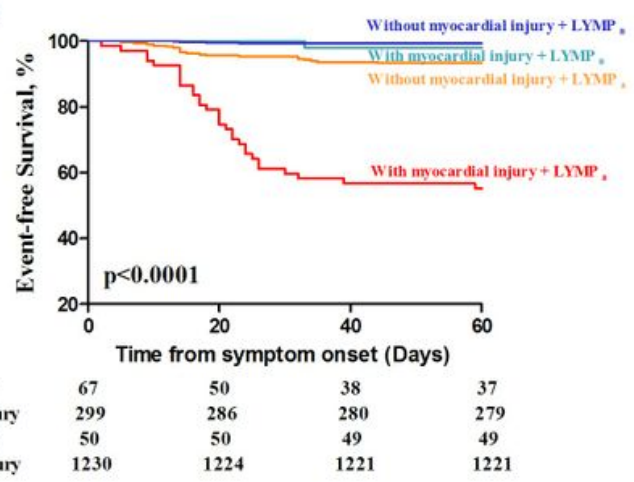

Figure 5

Event-free survival in COVID-19 patients according to myocardial injury and LYMP. Kaplan-Meier plot of survival rate (A), event-free (the incidence of severe cases) survival (B), event-free (the incidence of ARDS) survival (C), and event-free (ICU admission) survival (D) among patients with or without myocardial injury co-existed with LYMPa and LYMPn. LYMPa=abnormal lymphocyte percentage ( $<20$ or $>50 \%$ ). LYMPn=normal lymphocyte percentage (20-50 \%).

\section{Supplementary Files}

This is a list of supplementary files associated with this preprint. Click to download.

- Takehomefigure.tif

- Supplementtable.docx 